\title{
Remote Image and Leaf Reflectance Analysis to Evaluate the Impact of Environmental Stress on Grape Canopy Metabolism
}

N.S. Lang, ${ }^{1}$ J. Silbernagel, ${ }^{2}$ E.M. Perry, ${ }^{3}$ R. Smithyman, ${ }^{4}{ }^{\text {L. }}$. M ills, ${ }^{1}$ and R.L. Wample ${ }^{1}$

AdDITIONAL INDEX WORDS. Vitislabrusca, Concord, ultra violet radiation, photosynthesis, deficitwater stress

Summary. R esearch suggests that blackleaf (a leaf disorder in grape, Vitislabrusca L.) is induced by high levels of ultra violet ( U V) radiation and overall light intensity, resulting in color changes (purple-brown-black) for sun-exposed leaves of the outer canopy, and a corresponding $>50 \%$ reduction in photosynthesis. M etabolic indicators (photosynthesis and leaf water potential), percent blackleaf expression, and full spectrum leaf reflectance were mapped within vineyards using global positioning system (G PS) and digital remotely-sensed images. E ach image and data record was stored as an attribute associated with a specific vine location within a geographical information system (GIS). Spatial maps were created from the GIS coverages to graphically present the progression of blackleaf across vineyards throughout the season. Analysis included summary statistics such as minimum, maximum, and variation of green reflectance, within a vineyard by image capture date. Additionally, geostatistics were used to model the degree of similarity between blackleaf values as a function of their spatial location. Remote-image analysis indicated a decrease in percent greenness of about $45 \%$ between July and August, which was related to a decrease in photosynthesis and an increase in blackleaf symptom expression within the canopy. Examination of full spectral leaf reflectance indicated differences at specific wavelengths for grape leaves exposed to U V or water-deficit stress. This work suggests that remote-image and leaf spectral reflectance analysis may be a strong tool for monitoring changes in metabolism associated with plant stress. 
To use site-specific management practices, producers must deal with variation which occurswithin anyfield, orchard or vineyard. M onitoring differences in plant environmental stress within a site, is a tool that determines needed inputs as well as a key component to implementation of site-specific management. D evelopment of methods to quantify how environmental stresses contribute to crop variation within a site, and thereby adversely impact crop productivity are needed to utilize this method.

Blackleaf isa leaf disorder that can occur in vineyards throughout the world, but commonly occurs in 'Concord' grapevines growing in the Columbia Basin, of central Washington. Due to its negative impact on fruit ripening and overall stress to grapevines, it has been identified as the top production/ physiology research priority in juice grapes in the northwestern U .S. ( $N$ orthwest Center for Small Fruit Research, 1997). The disorder is characterized by an initial discoloration (purple-brown-black) of sun-exposed leaves of the outer canopy. Visual symptoms are traditionally apparent on leaves on the outer canopy in late July and early August and may progress to successive canopy layers by harvest (September to 0 ctober). In extreme cases, the entire canopy can be affected, resulting in defoliation of the vine. Blackleaf was reported to be related to low petiole and/ or leaf potassium concentration (Clore and Woodbridge, 1963; Shaulis, 1954), although the correlative relationship was highly variable. Tremendous differences in symptom expression between blackleaf and classic potassium deficiency in grape occur not only in terms of timing and location of leaf symptoms, but also in location of symptom expression within the canopy and on individual shoots ( $M$ alstrom, 1970). Blackleaf has been recently related to light dependent loss of chlorophyll and $>50 \%$ reduction in photosynthetic rate (Lang et al., 1998; Smithyman, 1999), thus diminishing the capacity of blackleaf-affected leaves to produce sugars and ripen fruits. Additionally, recent work has verified that leaf potassium contents are within or above recommended levels in blackleaf-affected vines (Smithyman, 1999), adding further evidence to discount the existing dogma. Instead, a significant seasonal decline in chlorophyll was found, suggesting permanent damage to the photosystem ( $L$ ang et al., 1998). In continuing studies, blackleaf symptom development was most strongly associated with a combination of high light intensity, U V-B irradiation, and water deficit (Smithyman, 1999). With more than 20,000 acres $(8,100$ ha) of 'Concord' currently under production in the Columbia Basin, blackleaf is a disorder which negatively influences the yield and quality of 'Concord' grapes in the largest juice production area within the U nited States. Characterization of the intensity and the spatial variation of blackleaf symptoms within and between vineyards would be required to objectively assess blackleaf impact on vine productivity within and among vineyards. Before the present work, no objectivemethod was established for quantifying thedistribution of blackleaf in a specific vineyard. Thus, determining the impact of blackleaf in the Columbia Basin production area wasnot economically possible due to the amount of acreage to be evaluated and the variation in severity of symptom expression within and among different vineyards.

Based on the highly visible symptomsasso ciated with blackleaf, the disorder appeared to have excellent potential as a model for integrating sitespecific horticulture management of canopy quality, with remote sensing and GPS technology. I mages collected from a point not in direct contact with an object of interest (remotely sensed images) provide a broad look at patterns which are difficult to decipher at field level, due to the scale on which they occur. Remotely sensed images can becollected from a variety of elevations (platforms) including: passive aerial devices (kite or helium blimp); fixed-wing aircraft at different altitudes; or satellites, depending on the appropriate scale and technical needs of the analysis ( $\mathrm{H}$ arris et al., 1996; Wilkie and Finn, 1996). In the work presented here, remotely sensed image capture was combined with geostatisitcal analysis to examine the directional trends and spatial dependency of blackleaf in two vineyards throughout the 1998 growing season. Additionally, a goal of this analysis was to further exploration of the spatial relationship of blackleaf to early season environmental stress patterns within the vineyard, to provide a means for growers to monitor and quantify the disorder, before visual symptom development. $C$ apture of remotely sensed imagery, has potential to provide real time information to identify variation within the production site. Timeliness of stress detection is needed for coupling site specific management strategies with immediate alterations of inputs to prevent negative impacts on yield or quality. Application of site specific management inputs before symptom expression may then avoid adverse impact on both crop quality and yield, while reducing inputs to meet goals of environmentally sound stewardship of agricultural land.

\section{Materials and methods}

STUdY SITE DESCRIPTION AND DATA COLLECTION INCORPORATING GLOBAL POSITION TECHNOLOGY.D atawerecollected in the Yakima Valley of eastern Washington, from a commercial 'Concord' vineyard (Dorsey) and a 'Concord' research vineyard $(\mathrm{H}$ 6) located at the Washington StateU niversity, I rrigated Agricultural Research and Extension Center (IAREC), at Prosser, Wash. In each vineyard, two blocks of vineswere designated which received standard commercial nutrient and water management. E ach block contained a minimum of 50 vines, to insure adequate sampling for spatial analysis of each vineyard. At each vine to be sampled, geographic coordinates were collected using a Trimble Global Positioning System (Trimble $\mathrm{N}$ avigation $\mathrm{Ltd}$., Sunnyvale, Calf.). Coordinates were collected $[2.3 \mathrm{ft}(0.7 \mathrm{~m})$ real time and $1.6 \mathrm{ft}(50 \mathrm{~cm}) \pm 1 \mathrm{ppm}$ postprocessing accuracy] in the universal transverse mercator (UTM ) projection system.

Remote-Image CaPture. During the 1996 and 1997 growing seasons, remote-image capture was tested using helium blimp [ 50 to $140 \mathrm{ft}$ ( 15.3 to $42.7 \mathrm{~m}$ ) altitude] and fixed-wing aircraft $[1,000 \mathrm{ft}(305 \mathrm{~m})$ altitude] platforms using $35 \mathrm{~mm}$ color (Ektachrome and Kodachrome, Eastman Kodak C o., Rochester, N .Y.) and infrared (color and black-andwhite) slide film. The two image capture platforms did not provide necessary image replication or sufficient image resolution, respectively. Based on evaluation of these image capture tests, ground-based digital images were collected above each sample vine during the 1998 growing season. A four-wheel drivevehiclewasused to movethrough the vineyard and serve as a platform on 
Fig. 1. (top) Remotely captured digital image of 'C oncord' canopy with blackleaf disorder, standard $18 \%$ gray photographic card and black fabric. The latter two items were used for calibration of light intensity, hue, and canopy area within the image. (middle) I nduced blackleaf on 'C oncord' exposed to ultra violet light and the same image with an overlay of green leaf areas selected by SigmaScan image analysis computer software (bottom) which place a color layer (light green in this example) over all areas within the image which fall within the defined hue and saturation thresholds.

which to stand to capture images, with acamera (D-500L; O lympus, M elville, N.Y.) directly over the top of each canopy. I mages were captured at three key phenological stages: fruit set (late J une to early-July), veraison, or the beginning of fruit ripening (August), and harvest (September to $O$ ctober). I mages were archived for later analysis at the end of the growing season.

Ground-based metabolic data. Photosynthetic rate, xylem water potential and visual estimates of blackleaf development based on mesophyllic browning (M B) and epidermal discoloration (ED) were measured to examine the relationship between changesin plant metabolism and blackleaf development. $\mathrm{N}$ et $\mathrm{CO}_{2}$ exchange of fully expanded sun leaves, under ambient vineyard conditions, was measured using an infrared gas analyzer (model LCA-2; Analytical Development Co., $\mathrm{H}$ oddesdon, England). Vine water statuswasmeasured using a pressurebomb (Plant M oisture Stress I nstrument C 0. ., Corvallis, O re.) to determine xylem water potential. Fully expanded leaves were selected and xylem water potential was measured after tissue reached equilibrium, as described by Smithyman (1999). Blackleaf symptom development was assessed (Smithyman, 1999) on leavesremoved from thesamecanopy location at which photosynthesis and xylem water potential were measured. E ach leaf was examined under $10 \times$ magnification with illumination under the field of view. The percentage of M B and ED within five leaf blade positions were determined. As a check of visual assessment, blackleaf symptoms were also analyzed using digital images (D -500L ; Olympus) from 50 leaves using SigmaScan Pro 2.0 (SPSS Inc., Chicago, III.) imageanalysispackage. There was a strong linear relationship $\left(r^{2}>\right.$
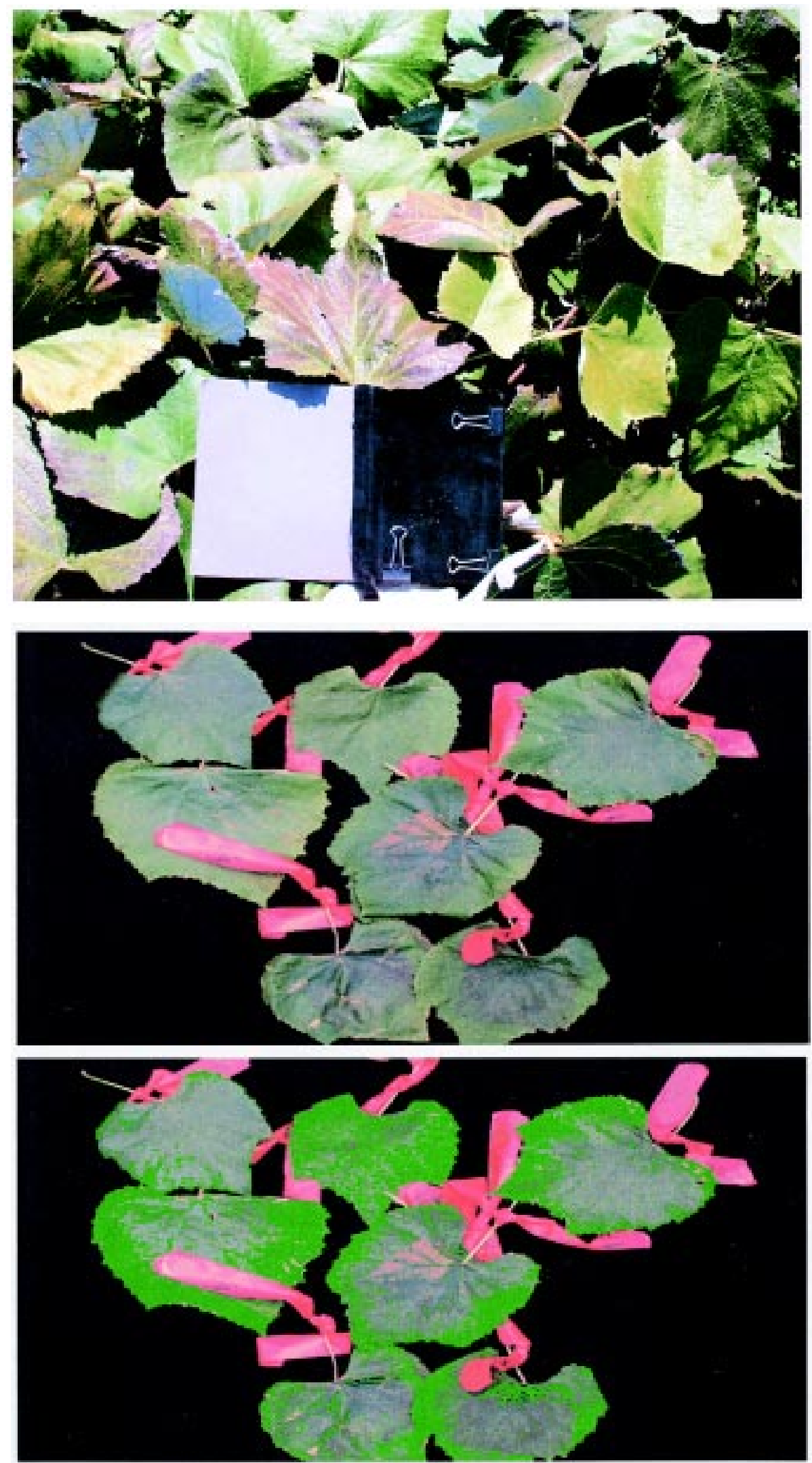

0.81 ) between visual and computer analyzed assessment of blackleaf. $\mathrm{Vi-}$ sual examinations of blackleaf (M B and ED ), photosynthetic rate and $x y-$ lem water potentials were not per- formed on the $\mathrm{H} 6$ vineyard due to limitations in equipment and personnel availability.

Ground-based portable spectroradiometric measurementswereused to 

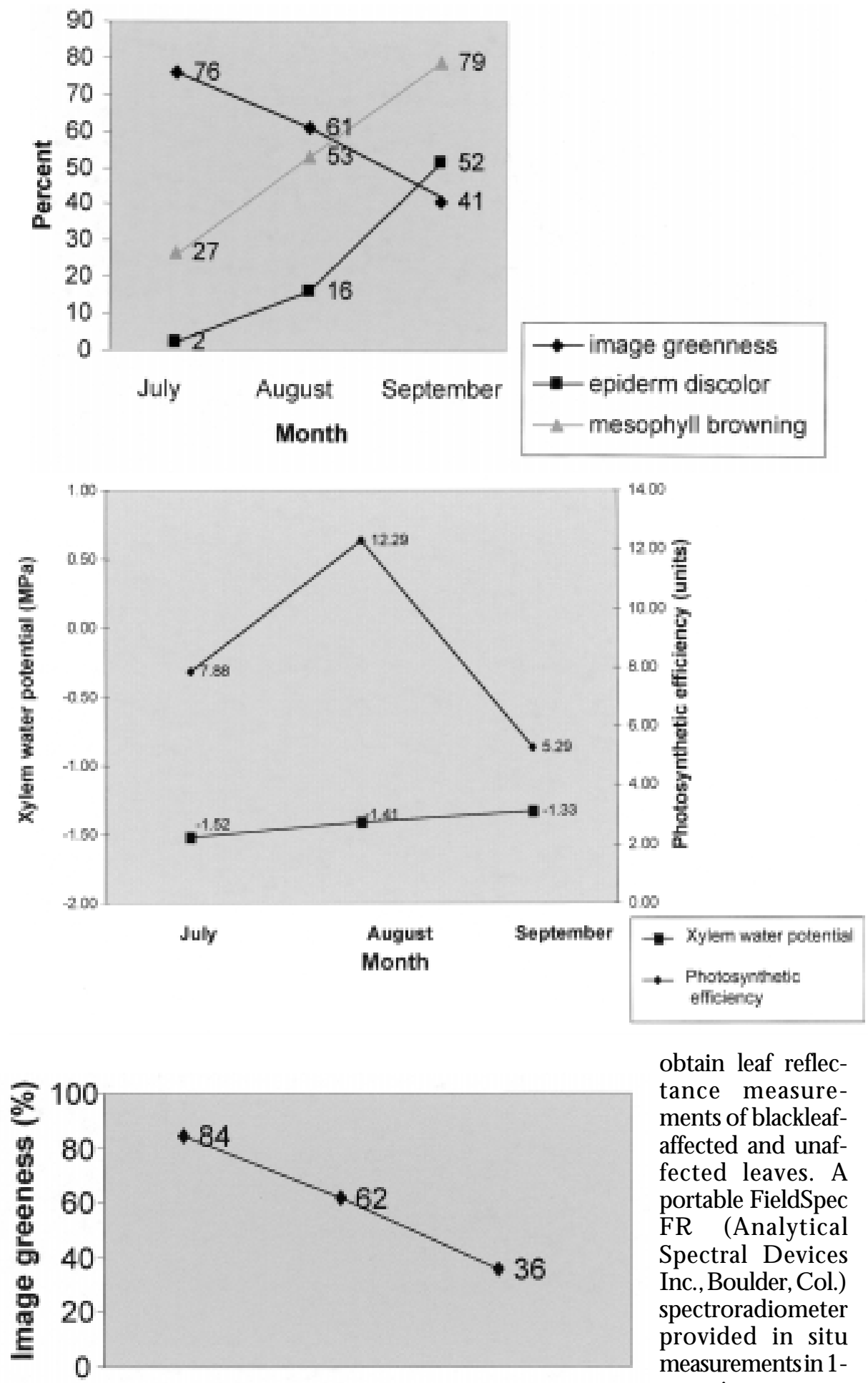

July

August September

Month

Fig. 2. Percent image greenness, epidermal discoloration, and mesophyll browning (top) and xylem water potential and photosynthetic efficiency (middle) in the 'C oncord' (Vitis labrusca ) commercial vineyard (Dorsey) during the 1998 growing season. Percent greenness in the research vineyard during the 1998 growing season (bottom). obtain leaf reflectance measurements of blackleafaffected and unaffected leaves. A portable FieldSpec FR (Analytical Spectral D evices Inc., Boulder, Col.) spectroradiometer provided in situ measurementsin 1$\mathrm{nm}$ increments from 450 to 2500 $\mathrm{nm}$ to create a full spectral curve library tor comparison with the corre sponding metabolic status of the grape leaf. Spectral libraries were analyzed to select a number of optimum bands (parameters derived from the bands) that best fit the range of plant conditions associated with plant stress indicators. Based on evidence that blackleaf symptom development (MB and ED) was closely associated with U V-B and water-deficit stress (Smithyman, 1999), leaves from greenhouse grown 'Concord' plants maintained under well watered or deficit-water conditions, were exposed to continuous or sequential durations of U V-B irradiation ranging from 10 to $30 \mathrm{~min}$ (10 $\mathrm{min} ; 10+10$ min; 20 min continuous exposure; 10 + $10+10 \mathrm{~min}$; and $30 \mathrm{~min}$ continuous exposure). Spectroradiometric measurementsof theseleaveswere used to determine whether unique spectral reflectance curvescould beidentified in order to differentiate one stressfrom theother under greenhouse conditions.

IMAGE ANALYSIS. Individual digital canopy images were analyzed using SigmaScan Pro 4.00.23 (SPSS Inc., Chicago, I II.) commercial image analysis software. To calibrate distance and area within each image, a $10 \times 20 \mathrm{~cm}$ (4 $\times 8$ inch) standard photographic gray card (18\% gray, D eltal/ CPM , D allas, Texas), with half the area covered by a flat black cloth was used. The gray/ black card waslaid on top of the canopy to beincluded in each image (Fig. 1). In cases in which insufficient card area was visible for calibration, image area was measured in pixel unitsrather than square centimeters.

U sing the SigmaScan D efine by Color tool, all areas of green leaves within the image were selected. Green wasused dueto thesimilarity of blackleaf discoloration to shadow, stems, or dark earth. Thus, using green provided greater accuracy in determining all leaf area which remained green (percent greenness) and allowed for calculation of the decrease in greenness through the season, as blackleaf developed. Preliminary color calibrations and threshold tests were completed on several sample images to determine correct hue and saturation levels, so as to consistently separate leaf greenness from the rest of the image. For images which may have had significantly different lighting characteristics, due to changes in solar radiation during image capture, hue and saturation were re-calibrated by comparison of the calibration card between theimageand all others. TheSigmaScan $D$ efineby Color tool placed acolor layer over all areas within theimage which fell within the defined hue and saturation thresholds (Fig. 2A and B). Selected threshold areas were visually verified to insure the layer adequately captured leaves and not areas within the images which were not of interest. The 


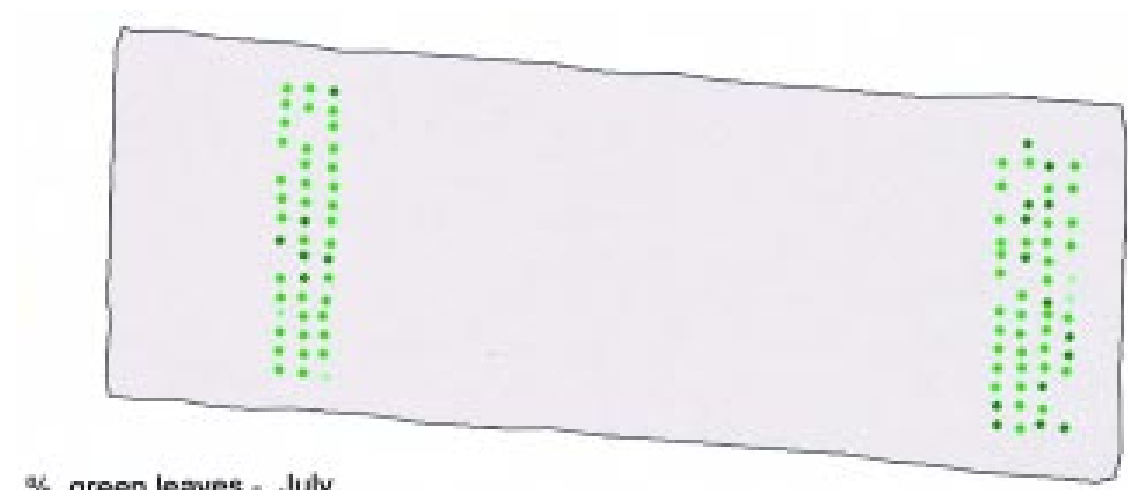

\% green leaves - July
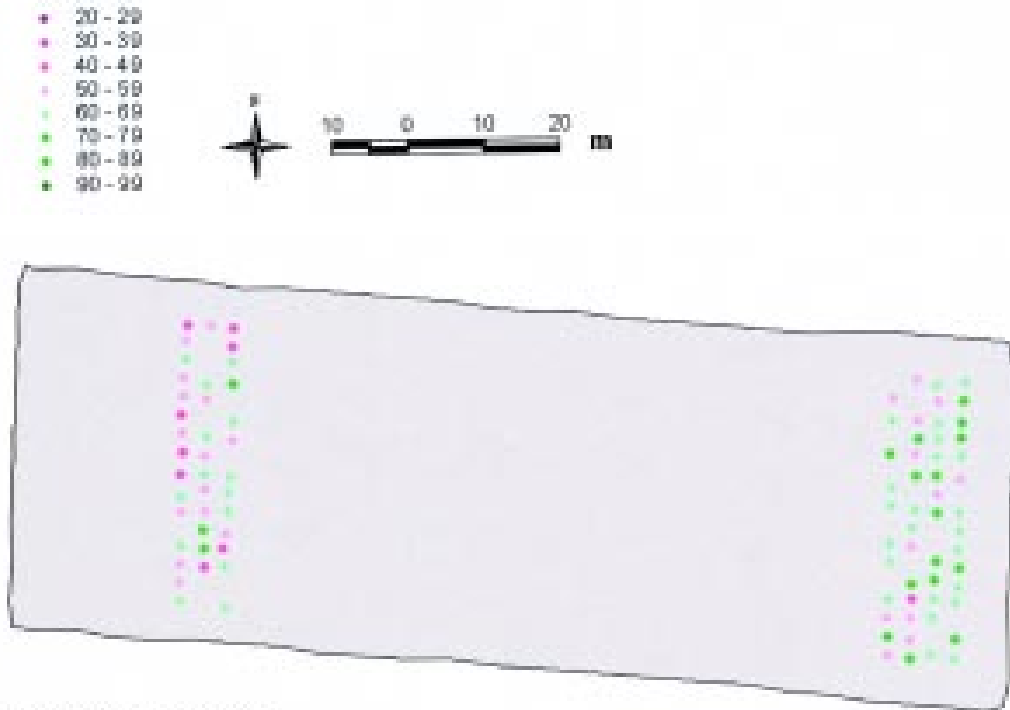

$\%$ green leaves - August
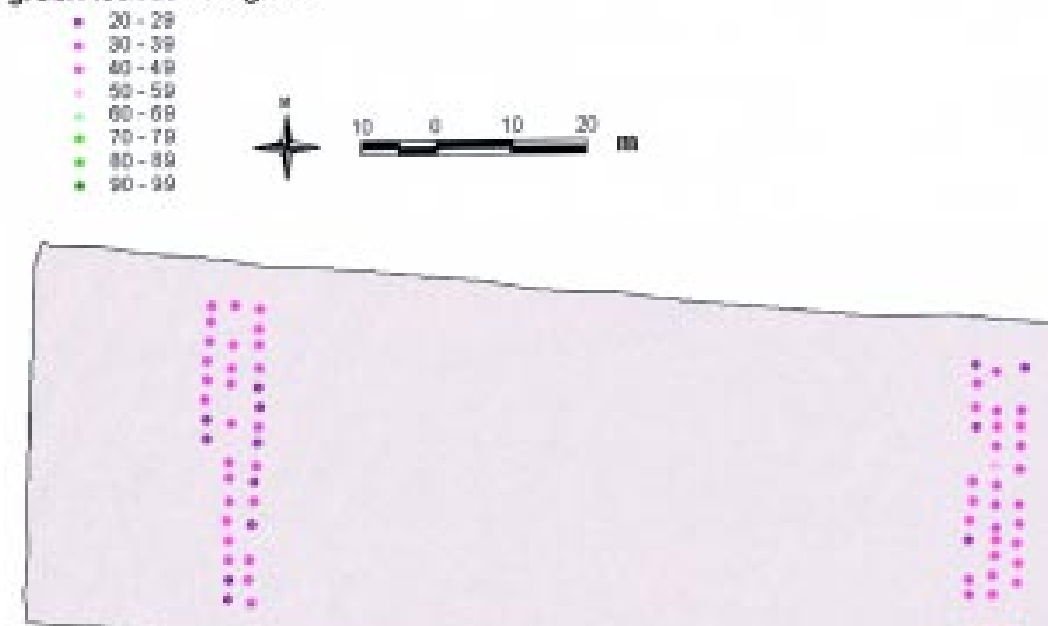

green leaves - September
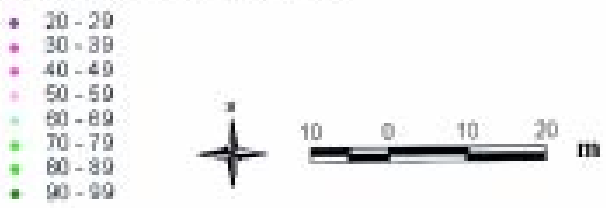

Fig. 3. Spatial maps of percent greenness in the ' $C$ oncord' research vineyard (H 6) for July (top), August (middle), and September (bottom) during the 1998 growing season created from analysis of ground-based digital images. Distance is reported in meters $(1 \mathrm{~m}=3.3 \mathrm{ft})$.
SigmaScan $M$ easure $D$ efined tool was used to cal culate defined threshold, calibration card, and total image areas. Percent greenness was calculated as \% green $=100 \times$ [area of green leaves/ (total image area - card area)].

Statistical analysis. Summarystatistics (minimum, maximum, average, $\mathrm{SE}$, variance and skew) were calculated by vineyard and sampling dates for a total of six data sets (two vineyards, three sampling dates each) to examine the development and variation of blackleaf (measured by decline in percent greenness) over the season. The decline in percent greenness was also compared with ground verification data already described. Spatial maps of percent greenness were created for each vineyard and sampling date using the GPS coordinates of each sampled vine and Arc/ Info 7.2.1 Geographic Information System ( Environmental SystemsR esearch I nstitute, I nc., R edlands, C alif.) to graphically represent progression of blackleaf visual symptom development throughout the season. Each image number was associated with $a(X$, Y) coordinate pair, with its respective percent greenness value as an attribute within the GI Scoverage. D atafileswere imported to Arc/ Info Generate, allowing the creation of a coverage map of each vineyard which was defined for UTM projection (ProjectD efine). ArcView GIS 3.1 (Environmental SystemsR esearch I nstitute, I nc., R edlands, C alif.) software was used to display and map the resulting coverages of percent greenness by sampling date for each vineyard. Variowin (PublicD omain Version 2.2) geostatistics software (Pannatier, 1996) was used to create variogram surfaces to model the degree of similarity between blackleaf symptoms, as a function of their spatial location. Directional trends (anisotrophy) were tested in all directions (omnidirectional) and in the direction of the long axis of the sample block. L ag spacings of 6.6 to $9.8 \mathrm{ft}(2$ to $3 \mathrm{~m}$ ) provided the resolution needed to examine the spatial structure of the data.

\section{Results and discussion}

$\mathrm{H}$ andheld, above canopy, digital images provided the necessary resolution to indicate distinct areas of visual blackleaf symptom expression by ED on individual leaves within each canopy (Fig. 1). ED symptom expression was visible within a few days of veraison (15 Aug. 1998). In the D orsey vine- 

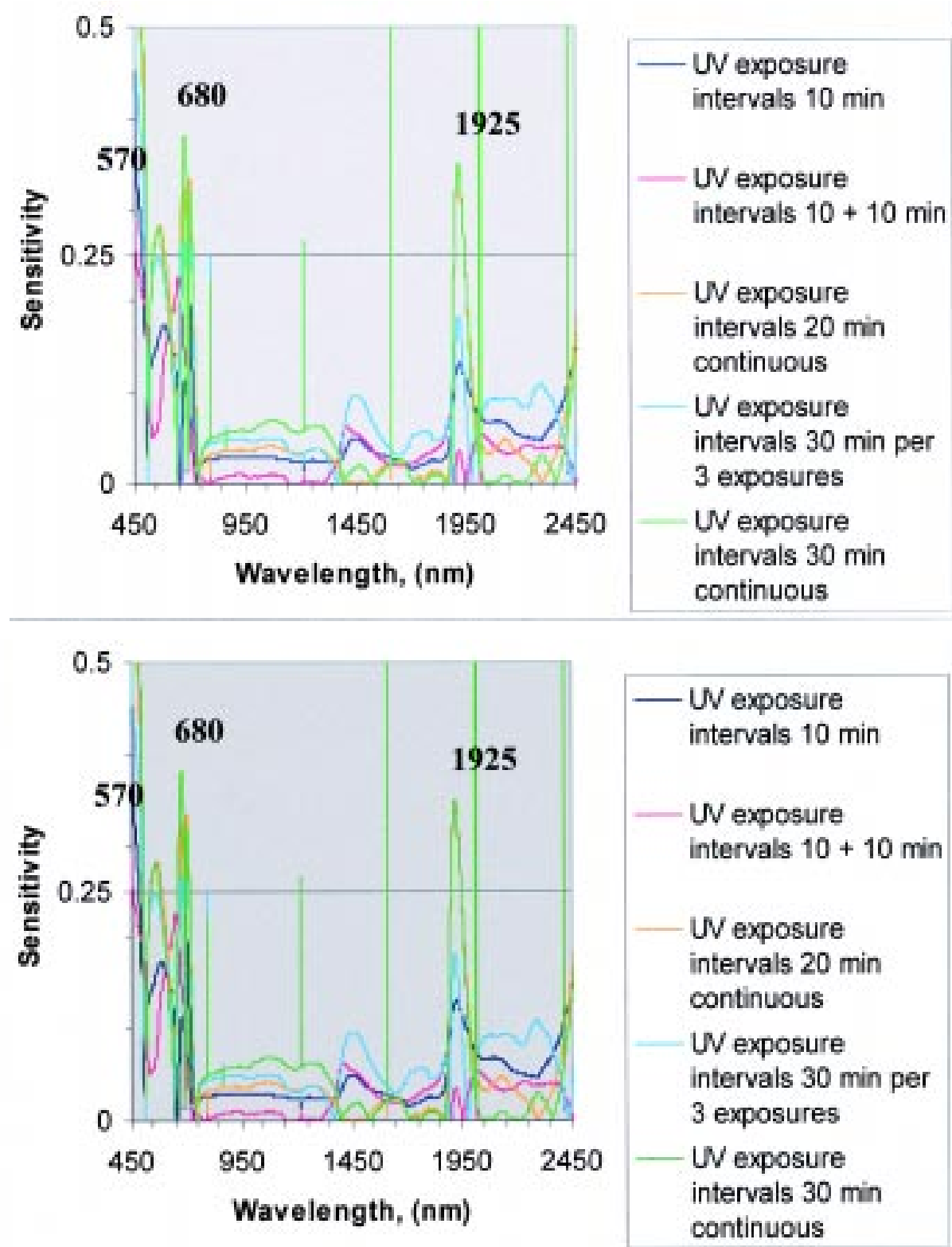

Fig. 4. F ull spectroradiometric leaf reflectance curves ('C oncord') from 450 to $4250 \mathrm{~nm}$ of leaves from greenhouse grown plants maintained at water-deficit (top) status or well-watered (bottom) and exposed to $10 \mathrm{~min}, 10+10 \mathrm{~min}, 20$ min continuous, $\mathbf{1 0}+\mathbf{1 0}+\mathbf{1 0}$ min or $\mathbf{3 0}$ min continuous ultra violet (UV) radiation. The difference in leaf reflectance between control and $U V$ and/or water-deficit plants is reported as sensitivity and are not presented with other units. D ifferences in leaf reflectance for water-deficit + UV radiation (top) grape plants were significant at 580, 670, 1420 and $1933 \mathrm{~nm}$. Differences in leaf reflectance for well-watered + U V radiation (bottom) grape plants were significant only at 570, 680, and $1925 \mathrm{~nm}$.

yard, M B was visible under 10× magnification several weeksbeforeED (Fig. $3 A)$. By harvest in September, M B and ED had affected $79 \%$ and $52 \%$ of the canopy in the D orsey vineyard, respectively. In comparison, the peak in photosynthetic rate occurred in August near veraison (Fig. 3B). During the same interval water potential declined (Fig. 3B ). L owest photosynthetic rates maps, created by analysis of groundbased digital images, the progression of blackleaf visual symptom development over the 1998 season was apparent. Early in the season (July), the digital images indicate a threshold quantity or area of green canopy for the $\mathrm{H} 6$ vineyard, within the field-ofview of the digital camera (Fig. 5A). Spatial maps of percent greenness in $\mathrm{H} 6$, based on analysis of ground-based digital images, indicated that within the $\mathrm{H} 6$ vineyard there was a progressive loss of greenness from July to August (Fig. 4A and B). Percent greenness significantly decreased by $35 \%$ and $48 \%$ from July to August 1998 in the D orsey and $\mathrm{H} 6$ vineyards, respectively (Fig. $3 \mathrm{~A}$ and $\mathrm{C}$ ). Therefore, the amount of green area within the field of view was significantly reduced by veraison, during a timeinterval in which green canopy area would be expected to increase (Fig. 5B). Blackleaf affected canopy area continued to increase between August and September 1998, with clearly visibleloss of canopy greenness (Fig. 4B and C). By harvest (September), well before normal leaf senescence, canopy greenness essentially disappeared (Fig. 5C). For the D orsey vineyard, loss of greenness, as quantified by digital image analysis, was correlated with a $53 \%$ and $49 \%$ increase of $M B$ and $E D$, respectively (Fig. 3A). The decline in greenness in the $D$ orsey vineyard al so corresponded to between a $33 \%$ to $57 \%$ decrease in photosynthesis by September when compared to photosynthetic rates in July and August, respectively (Fig. 3C).

Previouswork suggested that full spectroradiometric leaf reflectance measurements could be used to differentiate blackleaf-affected and unaffected 'C oncord' leaf tissue (Perry et al., 2000). Full spectroradiometric leaf reflectance measurements indicated distinct changes at specific wavelengths for well-watered +U V-irradiated leaves $(570,680$, and $1925 \mathrm{~nm})$ which were similar to water-deficit + U V-irradiated leaves $(580,670,1933 \mathrm{~nm}$ ) (Fig. $5 A$ and $B$ ), both of which were analyzed as a comparison to non-stressed plants (reported as sensitivity). An additional difference in leaf spectral reflectance was measured for leaves of water-deficit + U V-irradiated at the $1420 \mathrm{~nm}$ wave length, that did not occur in the leaf reflectance curves of control or well-watered + U V-irradiated plants (Fig. 5A and B). Thus, 
these data suggest the two imposed stresses of U V-irradiation and waterdeficit stress do not produce identical stress spectral reflectance curves, but rather could be separated by unique spectral signatures.

Spatial analysis of blackleaf development in the Dorsey and $\mathrm{H} 6$ vineyards indicated distinct spatial structure early in the season. Analysis of combined sampleblocks of the D orsey vineyard exhibited $39 \%$ of leaf greenness in July was dependent on location within the vineyard. In August, only the northeast block still had a spatial dependency of 38\%. By September little spatial dependency ( $13 \%$ to $17 \%$ ) could befound in either sample block of the vineyard. For the H 6 vineyard spatial dependency of the two sample blocks ranged from 33\% to $48 \%$ in July. As with the Dorsey vineyard, percent greenness in the $\mathrm{H} 6$ vineyard lost much of its spatial dependency in August. This suggests that the loss in spatial dependency of visual blackleaf symptom expression may in fact be the result of the disorder's pervasiveness throughout the vineyard by September. H owever, by September in the $\mathrm{H} 6$ vineyard the spatial dependency of the combined sample blocks in $\mathrm{H} 6$ was $49 \%$ Thus additional analysis of the spatial relationship of blackleaf in 'C oncord' vineyards will be necessary.

\section{Conclusion}

From this preliminary work we suggest that leaf spectral reflectancemayin fact beastrong tool for monitoring changesin metabolism associated with environmental stress. Geostatistics and spatial analysis of remote images appear to partially explain the variation of blackleaf symptom expression within vineyards early in the season, which is a critical time for assessment of plant stress. Continuing work will emphasize the testing of the unique leaf reflectancesignaturesasindicators of environmental stress, before visual symptom expression. The ability it pinpoint early season stress, when changes in vineyard management can alleviate stress conditions which result in negative impacts on fruit quality and yield holds potential as a key to the implementation of site-specific management practices. Continuing work within thissystem will focus on long-term monitoring of multiple locations to directly link biotic and abiotic plant stress (within the vineyard) with changes in canopy metabolism that influence overall canopy quality. Additionally, these techniques could monitor and detect changes in plant status over time within other site specific crop management systems, which may vary in scale from large field and orchard production to smaller production systems in nurseries and greenhouse operations. Such techniques hold potential for uses in the research and management of other ecological and physiological aspects, in natural ecosystems and production systems. By exploring remotesensing platforms which broaden the spatial content within image captureand/ or spectral reflectance measurements, it would be possibleto examinecommunity dynamics and the impact of agricultural operations on those ecosystems.

\section{Literature cited}

Clore, W.J . and C.G. Woodbridge. 1963. Someleaf abnormalities of C oncord grapes. Proc. Wash. State Hort. Assn. 59:181182.

H arris, N .R., D .E.J ohnson, T .L. Righetti, and M.R. Barrington. 1996. A blimp borne camera system for monitoring rangelands, riparian zones, or critical areas. Geocarto Intl. 11:99-104.

Lang, N.S., R.L. Wample, R. Smithyman, and L. Mills. 1998. Photosynthesis and chlorophyll fluorescence in blackleaf-affected Concord leaves. Amer. J. Enol. Viticult. 49:367-373.

M alstrom, H .L. 1970. Some factors leading to the production of pigmentsin leaves of Vitis. PhD diss. U niv. Calif., D avis.

N orthwest Center for Small F ruit Research. 1997. Production/ physiology research priorities. Proc. NW Ctr. Small Fruit Res. 6:13.

Pannatier, Yvan. 1996. Variowin: Software for spatial data analysis in 2D. SpringerVerlag, N ew York.

Perry, E.M., S.Lang, J. Davenport, D.N . Anderson, and K.H. J armon. 2000. Spectral reflectance monitoring of crop stress. 2nd I ntl. Conf. Geospatial I nfo. Agr. For. 1:344-350.

Shaulis, N. 1954. Potash deficiency in the vineyard and its cure. N.Y. Farm Res. 20:4.

Smithyman, R.P. 1999. Environmental factors affecting the physiology and development of blackleaf in Concord grapevines. PhD diss., Wash. State U niv., Pullman.

Wilkie, D.S. and J.T. Finn. 1996. Remote sensing imagery for natural resourcesmonitoring. Columbia U niv. Press, N ew York. 